OPEN ACCESS

Edited by:

P. Ashley Wackym,

The State University of New Jersey,

United States

Reviewed by:

Daniel J. Lee,

Massachusetts Eye \& Ear Infirmary and Harvard Medical School,

United States

Denise Utsch Gonçalves,

Federal University of Minas

Gerais, Brazil

${ }^{*}$ Correspondence:

Laura Fröhlich

laura.froehlich@uk-halle.de

Specialty section:

This article was submitted to

Neuro-Otology,

a section of the journal

Frontiers in Neurology

Received: 07 April 2020 Accepted: 22 September 2020

Published: 27 October 2020

Citation:

Fröhlich L, Curthoys IS, Kösling S, Obrist D, Rahne T and Plontke SK

(2020) Cervical and Ocular

Vestibular-Evoked Myogenic

Potentials in Patients With

Intracochlear Schwannomas.

Front. Neurol. 11:549817.

doi: 10.3389/fneur.2020.549817

\section{Cervical and Ocular Vestibular-Evoked Myogenic Potentials in Patients With Intracochlear Schwannomas}

\author{
Laura Fröhlich $^{1 *}$, Ian S. Curthoys ${ }^{2}$, Sabrina Kösling ${ }^{3}$, Dominik Obrist ${ }^{4}$, Torsten Rahne ${ }^{1}$ and \\ Stefan K. Plontke ${ }^{1}$ \\ ${ }^{1}$ Department of Otorhinolaryngology, Head and Neck Surgery, Martin Luther University Halle-Wittenberg, Halle (Saale), \\ Germany, ${ }^{2}$ Vestibular Research Laboratory, School of Psychology, The University of Sydney, Sydney, NSW, Australia, \\ ${ }^{3}$ Department of Radiology, Martin Luther University Halle-Wittenberg, Halle (Saale), Germany, ${ }^{4}$ ARTORG Center for \\ Biomedical Engineering Research, University of Bern, Bern, Switzerland
}

Objective: To evaluate ocular and cervical vestibular evoked myogenic potentials (oVEMPs and CVEMPs) in patients with solely intracochlear localization of an intralabyrinthine schwannoma (ILS).

Study Design: Retrospective analysis of a series of cases.

Setting: Monocentric study at a tertiary referral center.

Patients: Patients with intracochlear schwannoma (ICS) and VEMP measurements.

Outcome Measures: Signed asymmetry ratio (AR) of cVEMPs and oVEMPs to air conducted sound with AR cut-offs considered to be asymmetrical when exceeding $\pm 30 \%$ for cVEMPs and $\pm 40 \%$ for oVEMPs with respect to the side affected by the tumor (reduced amplitudes on the affected side indicated by negative values, enhanced amplitudes by positive values); VEMP amplitudes and latencies; tumor localization in the cochlear turn and scala.

Results: Nineteen patients with a solely intracochlear tumor (ICS patients) [10 males, 9 females, mean age 57.1 (SD: 13.4) years] were included in the study. On the affected side, cVEMPs were absent or reduced in $47 \%$ of the patients, normal in $32 \%$, and enhanced in $21 \%$. Ocular VEMPs on the affected side were absent or reduced in $53 \%$ of the patients, normal in 32\% and enhanced in 15\%. Latencies for cVEMPs and oVEMPs were not significantly different between the affected and non-affected side. In all patients with enhanced VEMPs, the tumor was located in the scala tympani and scala vestibuli.

Conclusions: As a new and unexpected finding, VEMP amplitudes can be enhanced in patients with intracochlear schwannoma, mimicking the third window syndrome.

Keywords: third window, vestibular schwannoma, intralabyrinthine, intracochlear, VEMP, asymmetry, secondary hydrops, semicircular canal dehiscence 


\section{INTRODUCTION}

It was observed that intralabyrinthine schwannomas (ILS) can mimic various common cochleovestibular diseases in their symptoms and findings in functional tests. Cochleovestibular schwannomas in general, often also referred to as vestibular schwannomas or acoustic neuromas, are benign tumors that arise from the Schwann cells of the eighth cranial nerve. The schwannomas are referred to as ILS, when they arise from the most peripheral branches of the cochlear or vestibular nerves, i.e., inside the membranous labyrinth (1). ILS can present e.g., with sudden, progressive, or fluctuating hearing loss, pseudo-conductive hearing loss, and/or vertigo, and/or (pulsating) tinnitus, and have been misdiagnosed e.g., as Menière's disease (MD) or sudden hearing loss (2-8). The diagnosis is based on high-resolution magnetic resonance imaging (MRI). Various slightly differing classifications of these tumors have been suggested in the literature $(2,5,9)$. The most recent and detailed classification was suggested by Van Abel et al. (5) distinguishing intracochlear, intravestibular, intravestibulocochlear, transmodiolar, transmacular, tympanolabyrinthine, translabyrinthine, and transotic locations or extensions. An extension from the internal auditory canal into the cerebellopontine angle is possible. Van Abel et al. (5) have also described that vertigo and imbalance were commonly reported when the tumors also extended to the vestibular labyrinth but were only reported by $36 \%$ of patients with intracochlear schwannomas (ICS). Intracochlear tumor localization seems to be the most common in $\operatorname{ILS}(2,9)$.

The recording of cervical and ocular vestibular evoked myogenic potentials (cVEMPs, oVEMPs) has been described as a screening tool for the assessment of nerve of origin in patients with cochleovestibular schwannoma (10). However, only few data are available on VEMPs in patients with an ILS. Lee et al. (11) described absent or decreased cVEMPs and oVEMPs in patients with ILS without specifying the exact tumor location. Ralli et al. (12) reported an absent oVEMP in a patient with an intravestibular ILS with presence of "a solid mass in the utricle" confirmed by magnetic resonance imaging (MRI). Dubernard et al. (6) analyzed cVEMPs in $36(32 \%)$ of their reported 110 patients with ILS. Twelve patients had an intracochlear tumor and cVEMPs were abnormal, i.e., absent or significantly reduced, in $50 \%$ of these patients and preserved in the remaining $50 \%$. To date, there is no study in which both oVEMPs and cVEMPs were systematically analyzed in a series of patients with solely intracochlear tumors.

VEMPs have also been described to be highly sensitive to changes in the inner ear fluid dynamics and to detect defects of the bony labyrinthine wall. In 1998, Minor et al. (13) were

\footnotetext{
Abbreviations: ILS, intralabyrinthine schwannoma; MD, Menière's disease; MRI, magnetic resonance imaging; ICS, intracochlear schwannoma; cVEMP, cervical vestibular evoked myogenic potential; oVEMP, ocular vestibular evoked myogenic potential; SSCD, superior semicircular canal dehiscence; VEMP, vestibular evoked myogenic potential; EMG, electromyogram; BT, basal turn; MT, middle turn; AT, apical turn; ST, scala tympani; SV, scala vestibuli; CT, computed tomography; AR, asymmetry ratio; AS, affected side; NAS, non-affected side; ICC, intraclass correlation coefficient.
}

the first to report about patients with a defect in the bony wall of the superior semicircular canals, a superior semicircular canal dehiscence (SSCD). Over time, various conditions with a defect of the labyrinthine bony wall have been described in the literature (14-22). These are associated with a similar spectrum of symptoms and objective findings so that these conditions are now summarized under the general term of "third window syndrome" or "third window abnormalities" $(23,24)$. Wackym et al. (24) defined the following conditions associated with the term of third window syndrome: "SSCD, cochlea-facial nerve dehiscence, cochlea-internal carotid artery dehiscence, cochlea-internal auditory canal dehiscence, lateral semicircular canal-superior semicircular canal ampulla dehiscence, modiolus, perilymph fistula, posterior semicircular canal dehiscence, posterior semicircular canal-jugular bulb dehiscence, SSCDsubarcuate artery dehiscence, SSCD-superior petrosal vein dehiscence, vestibule-middle ear dehiscence, lateral semicircular canal-facial nerve dehiscence, wide vestibular aqueduct in children, post-traumatic hypermobile stapes footplate, otosclerosis with internal auditory canal involvement." The objective findings of increased VEMP amplitudes and/or lower VEMP thresholds have been reported in patients with SSCD (25-28), posterior semicircular canal dehiscence $(16,29)$, large vestibular aqueduct (30), perilymph fistula (31), cochlea-facial nerve dehiscence (24), posterior semicircular canal-jugular bulb dehiscence (32), and SSCD-superior petrosal vein dehiscence (33). In these patients, the presence of a third window caused by an otic capsule defect changed the mechanical properties, i.e., the fluid dynamics, of the inner ear. It has been shown by measurements and models, that in SSCD ears incoming acoustic energy causes larger fluid displacement in the semicircular canals (34-36). Furthermore, animal studies demonstrated that this results in activation of semicircular canal neurons in addition to otolith neurons (37). These canal afferents project to the contralateral (external ocular) inferior oblique muscle as well as the ipsilateral sternocleidomastoid muscle (inhibitory) and thus their activity contributes to and enhances cVEMPs and oVEMPs (38).

Apart from defects in the bony labyrinth, other inner ear pathologies have the potential to impact inner ear mechanics. Endolymphatic hydrops for instance, which can have various causes $(39,40)$, is believed to have a huge impact on inner fluid mechanics (41-43). This argument is supported by VEMP studies in patients suffering from clinically diagnosed definite MD. Asymmetric, enhanced cVEMPs $(44,45)$ but also enhanced oVEMPs in MD patients have been reported $(46,47)$. These inner ear pathologies can therefore mimic third window syndrome with regard to VEMP test results. While ILS can mimic other cochleovestibular diseases in their symptoms and functional findings, it is unknown, if ILS can also mimic third window syndrome as was described for other inner ear pathologies.

The aim of this study was to review and describe oVEMPs and cVEMPs of patients with solely intracochlear localization of an ILS. By including only ICS patients, we sought to avoid those with direct impact of the intravestibular schwannoma on otolith organs which might lead to a change in VEMP results. 


\section{MATERIALS AND METHODS}

\section{Study Design and Participants}

In this retrospective analysis, patients of a single tertiary referral center were included. In a personal case series (SKP) at the University Hospital Halle of 53 consecutive patients with intralabyrinthine schwannoma (ILS), magnetic resonance images were analyzed for localization of the tumor. Patients with solely intracochlear schwannoma (ICS) in whom VEMP measurements had been performed between August 2015 and January 2020 were included in this study (ICS patient group). Patients without VEMP measurements or with other tumor localizations [see introduction according to Van Abel et al. (5)] were excluded from this study to avoid influence by direct impact of the tumor on the otoliths or by retrocochlear pathology.

Written informed consent was obtained from all patients. The study protocol was reviewed and approved by the responsible institutional review board (ethics committee of the Medical Faculty of Martin Luther University Halle-Wittenberg and the University Hospital Halle, approval number: 2019-26), and conducted according to the Declaration of Helsinki.

\section{VEMP Testing}

The VEMP tests of the included patients were reviewed. All VEMP recordings were collected and analyzed using the Eclipse recording platform (Interacoustics A/S, Middelfart, Denmark). Self-adhesive Neuroline 720 surface electrodes (Ambu A/S, Ballerup, Denmark) were used for electromyogram (EMG) recording after the skin was prepared to provide impedances of $5 \mathrm{k} \Omega$ or less. For cVEMPs, the electrodes were placed over the middle of the sternocleidomastoid muscle ipsilateral to the stimulated ear and over the sternum. For oVEMP recordings, the electrodes were placed on the infra-orbital ridge $1 \mathrm{~cm}$ below the lower eyelid contralateral to the stimulated ear and about $2 \mathrm{~cm}$ below the first electrode. The ground electrode was always positioned on the forehead.

During VEMP testing the patients were sitting on a chair. They were asked to turn their head to the contralateral shoulder for cVEMP testing and hold this position to achieve a constant tonic activation of the sternocleidomastoid muscle $(50-200 \mu \mathrm{V})$ during the whole recording period. During data acquisition the EMG was monitored and appropriate feedback was provided in real time to ensure that sufficient muscular contraction was sustained (48). For oVEMP testing, the patients were asked to keep their head in a neutral position and look up, maintaining an angle of $20-30^{\circ}$.

For both, cVEMP, and oVEMP testing, air-conducted $500 \mathrm{~Hz}$ tone bursts ( 1 cycle rise/fall time, 2 cycles plateau) were delivered by ER-3A insert earphones (3M, St. Paul, MS, USA) at $100 \mathrm{~dB}$ nHL.

The EMG signals were recorded in a -20 to $80 \mathrm{~ms}$ window relative to the onset of the stimulus. A bandpass filter of 10$1,000 \mathrm{~Hz}$ was applied and the artifact rejection level was set to $400 \mu \mathrm{V}$. The responses were averaged to at least 200 stimuli and at least two trials were recorded for each VEMP test.

\section{Specifying Tumor Location}

All patients underwent MRI of the temporal bone with at least thin-sliced 3D T2-weighted and T1-weighetd images with contrast medium. In all included patients, the MRI was (retrospectively) systematically studied regarding the localization of the tumor. The classification suggested by Van Abel et al. (5) was used to classify tumor localization in the basal turn (BT), middle turn (MT), or apical turn (AT) of the cochlea, including combinations of these localizations. Additionally, localization of the tumor in the scala tympani (ST) and/or scala vestibuli (SV) was specified, if possible. The MRIs originated from different sources, often from outside our hospital, and thus, showed considerable differences in resolution. MRIs were not repeated, if the scans were sufficient for establishing the diagnosis of ICS.

\section{Exclusion of Third Window Syndromes}

Temporal bone computed tomography (CT) scans or cone beam CTs were retrospectively analyzed for the presence of semicircular canal dehiscence, enlarged vestibular aqueduct, cochlea-facial nerve dehiscence, and other third window syndromes [see introduction and (24)]. It has to be noted, however, that the intention for performing the CT and cone beam CT scans were solely for preoperative evaluation of the bony anatomy prior to a possible surgery for tumor removal and hearing rehabilitation with a cochlear implant. They were performed after the diagnosis of an ILS was established by MRI and thus they usually did not include specific reconstructions for evaluation of "third windows" of the inner ear, e.g., no planes along the superior semicircular canal.

The patients' medical histories taken at initial presentation including audiological and vestibular complaints were retrospectively evaluated for typical symptoms of third window lesions including vertigo or oscillopsia induced by loud sounds/Tullio phenomenon, increased sensitivity to low frequency sounds, autophony, and pulsating tinnitus.

\section{Data Analysis}

A VEMP was ultimately judged as present, when the putative response was clearly larger than the pre-stimulus waveforms, i.e., the background noise. The impact of muscle contraction on cVEMP results was reduced by averaging the root mean square of the EMG signal over the pre-stimulus window and for each recording frame to calculate the background EMG, i.e., the contraction strength.

The p13 n23 for cVEMPs and n10 p15 for oVEMPs were identified and peak latencies as well as peak-to-peak amplitudes were recorded. The p13 n23 peak-to-peak amplitude was normalized to the background EMG. The asymmetry ratio (AR) was calculated from the peak-to-peak amplitudes. In order to account for the side affected by the tumor (AS) and the nonaffected side (NAS) and to overcome the drawback of absolute $\mathrm{AR}$, a signed AR was used:

$$
A R(\%)=\frac{\text { amplitude }(A S)-\text { amplitude }(N A S)}{\text { amplitude }(A S)+\text { amplitude }(N A S)} * 100 .
$$

For cVEMPs, the AS refers to the response recorded from the ipsilateral sternocleidomastoid muscle and for oVEMPs the AS 
refers to the response recorded from the contralateral inferior oblique muscle. For cVEMPs, ARs above $30 \%$ or below $-30 \%$ were considered abnormal (49). For oVEMPs, abnormal ARs were above $40 \%$ or below $-40 \%(50,51)$. Positive values of the AR indicate larger responses of the affected ear (enhanced), while negative values indicate smaller responses of the affected ear (reduced), respectively. If no response could be detected, the amplitude was set to $0 \mu \mathrm{V}$. For unilateral responses, the AR was therefore $100 \%$ or $-100 \%$.

VEMP analysis was performed by two blinded examiners. Normal distribution of the amplitude and latency data was confirmed by a Shapiro-Wilk test. The intraclass correlation coefficient [ICC $(3,1)]$ was calculated for the oVEMP and cVEMP ARs based on the analysis by the two examiners to assess the inter-rater reliability. If no responses could be detected on the AS and NAS, the AR was set to $0 \%$ for the statistical test (see following paragraph). Inter-rater agreement was considered "poor" for ICCs below 0.50, "moderate" between 0.50 and 0.75 , "good" between 0.75 and 0.90 , and "excellent" above 0.90 (52). Good or excellent agreement was considered acceptable for further analysis. The final latencies and amplitudes were the averages of the examiners. For absence of a response rated by one examiner but presence of a response rated by the other examiner, the amplitudes were the averages and the latencies were taken from the one examiner who rated the response to be present. The cVEMP and oVEMP latencies and amplitudes recorded from the AS were compared to the responses from the NAS as control by paired $t$-tests. A confidence level of $95 \%$ or above was considered to be significant $(p<0.05)$. SPSS statistics (IBM, Armonk, New York, USA) was used for all statistical analyses.

The VEMP results were related to tumor localization in a hypotheses generating descriptive analysis.

\section{RESULTS}

Twenty-six patients with solely intracochlear schwannoma (ICS) were identified. Six patients had not undergone VEMP testing and were therefore excluded. The analysis of the CTs or cone beam CTs (available in 16 patients) revealed a dehiscent superior semicircular canal in one case. This patient was excluded as well. There were no signs for other third mobile windows of the otic capsule. Thus, 19 patients with ICS were included in the study for final analyis. Of those, 10 patients were male, 9 were female. The mean age was 57.1 (SD: 13.4) years. In 8 patients, the left ear was affected, in 11 patients the tumor was located in the right ear. The mean hearing threshold [pure tone average at $0.5,1,2$, and $4 \mathrm{kHz}$ (4PTA)] was 90.8 (SD: 25.0) dB HL for the affected side (AS) and 18.7 (SD:13.3) dB HL for the non-affected side (NAS). Some of the patients reported pulsating tinnitus and very few patients reported autophony and increased sensitivity for low frequency sounds. Other typical clinical symptoms of third window lesions like oscillopsia or vertigo induced by loud sounds/Tullio phenomenon have not been observed in any of those patients.

Despite the different image resolution of the MRIs, it was possible to specify tumor localization according to basal turn
(BT), middle turn (MT), and the apex (apical turn, AT) in all patients. Only in one patient, it was difficult to localize the tumor with respect to the scala tympani (ST), and/or scala vestibuli (SV). Data for all patients are summarized in Table $\mathbf{1}$. The tumor was located in the BT in 2 patients (11\%), in the MT in 7 patients (37\%), and in the AT in 1 patient (5\%). In 4 patients (21\%), the tumor was in the BT and MT, in 4 patients (21\%) it was in the MT and AT, and in 1 patient (5\%) it was in the BT, MT, and AT. With respect to the scalae, tumors were observed solely in ST in 5 patients (26\%). In no patient, the tumor was solely located in SV, and in 14 patients (74\%) it was located in both, ST, and SV.

Regarding the VEMP analysis, the inter-rater reliability analysis by ICC revealed good to excellent agreement between the two raters. For cVEMPs, the single measure ICC was 0.990 with a $95 \%$ confidence interval from 0.996 to $0.975\left[F_{(18)}=205.248\right.$, $p<0.001]$. For oVEMPs, the single measure ICC was 0.875 with a $95 \%$ confidence interval from 0.706 to $0.950\left[F_{(18)}=15.052, p\right.$ $<0.001]$. Cervical VEMPs could be recorded from the affected side (AS) in 13 cases (68\%). In 4 cases, the response was absent in the non-affected side (NAS) as well. In the other 2 cases, the AS was the only ear without a response (AR $=-100 \%)$. The oVEMP measurements showed responses of the AS in 10 patients (53\%). In 6 cases, it was absent in both, AS and NAS. In 3 cases, the AS was the only side without a response ( $A R=-100 \%)$. The mean p13 n23 cVEMP amplitude was 0.46 (SD: 0.52) for the AS and 0.50 (SD: 0.45) for the NAS. For oVEMPs, the mean n10 p15 amplitude was $2.44 \mu \mathrm{V}$ (SD: $4.27 \mu \mathrm{V}$ ) for the AS and $1.89 \mu \mathrm{V}$ (SD: $2.10 \mu \mathrm{V}$ ) for the NAS. The mean cVEMP p13 latencies were $16.5 \mathrm{~ms}$ (SD: $1.8 \mathrm{~ms}$ ) for the AS and $16.1 \mathrm{~ms}$ (SD: $1.7 \mathrm{~ms}$ ) for the NAS, mean n23 latencies were $26.1 \mathrm{~ms}$ (SD: $2.9 \mathrm{~ms}$ ) and $25.6 \mathrm{~ms}$ (SD: $2.2 \mathrm{~ms}$ ), respectively. For oVEMPs, the mean n10 latencies were $12.7 \mathrm{~ms}$ (SD: $1.0 \mathrm{~ms}$ ) for the AS and $12.4 \mathrm{~ms}$ (SD: $0.8 \mathrm{~ms}$ ) for the NAS, and p15 latencies were $18.1 \mathrm{~ms}$ (SD: $1.3 \mathrm{~ms}$ ) and $17.9 \mathrm{~ms}$ (SD: $1.2 \mathrm{~ms}$ ), respectively. Between the AS and NAS, no significant difference was found for $\mathrm{p} 13$ and $23 \mathrm{cVEMP}$ latencies $\left[t_{(12)}=1.267, p=0.229 ; t_{(12)}=1.216, p=0.247\right]$ as well as for the $\mathrm{n} 10$ and $\mathrm{p} 15$ oVEMP latencies $\left[t_{(9)}=1.552, p=0.155 ; t_{(9)}=\right.$ $0.998, p=0.344]$. The results are illustrated in Figure 1A.

The VEMP asymmetry ratio (AR) results are given for each patient in Table 1. Figure 1B illustrates the results in a boxplot. Patients are color coded as they contribute to both the oVEMP and cVEMP AR data. If no response could be recorded on both the AS and NAS, the AR was illustrated at $0 \%$ by an empty circle in the plot. The mean AR was - 15.6\% (SD: 53.6\%) for cVEMPs and $-15.3 \%$ (SD: $64.5 \%$ ) for oVEMPs. For cVEMPs, the AR was smaller than $-30 \%$, i.e., asymmetrical with reduced responses on the AS, in 5 cases (26\%), including the 2 cases with ARs of $-100 \%$. The AR was larger than $30 \%$, i.e., asymmetrical with enhanced responses in the AS, in 4 cases (21\%). Including the 3 cases with ARs of $-100 \%$, the oVEMP AR was smaller than $-40 \%$ in 4 cases (21\%) and larger than $40 \%$ in 3 cases (16\%). In total, VEMPs were enhanced on the AS in 5 patients: in 2 patients only the cVEMPs (\#8, \#19), in 1 patient only the oVEMPs (\#16), and in 2 patients both, the cVEMPs and oVEMPs (\#13,\#14). The VEMP results of these patients are illustrated in Figure 2.

CT or cone beam CT scans were available in four of five patients with enhanced VEMPs (patient \#8 declined the CT) 
TABLE 1 | Demographic data, OVEMP and cVEMP asymmetry ratio (AR) results, and tumor localization of included patients.

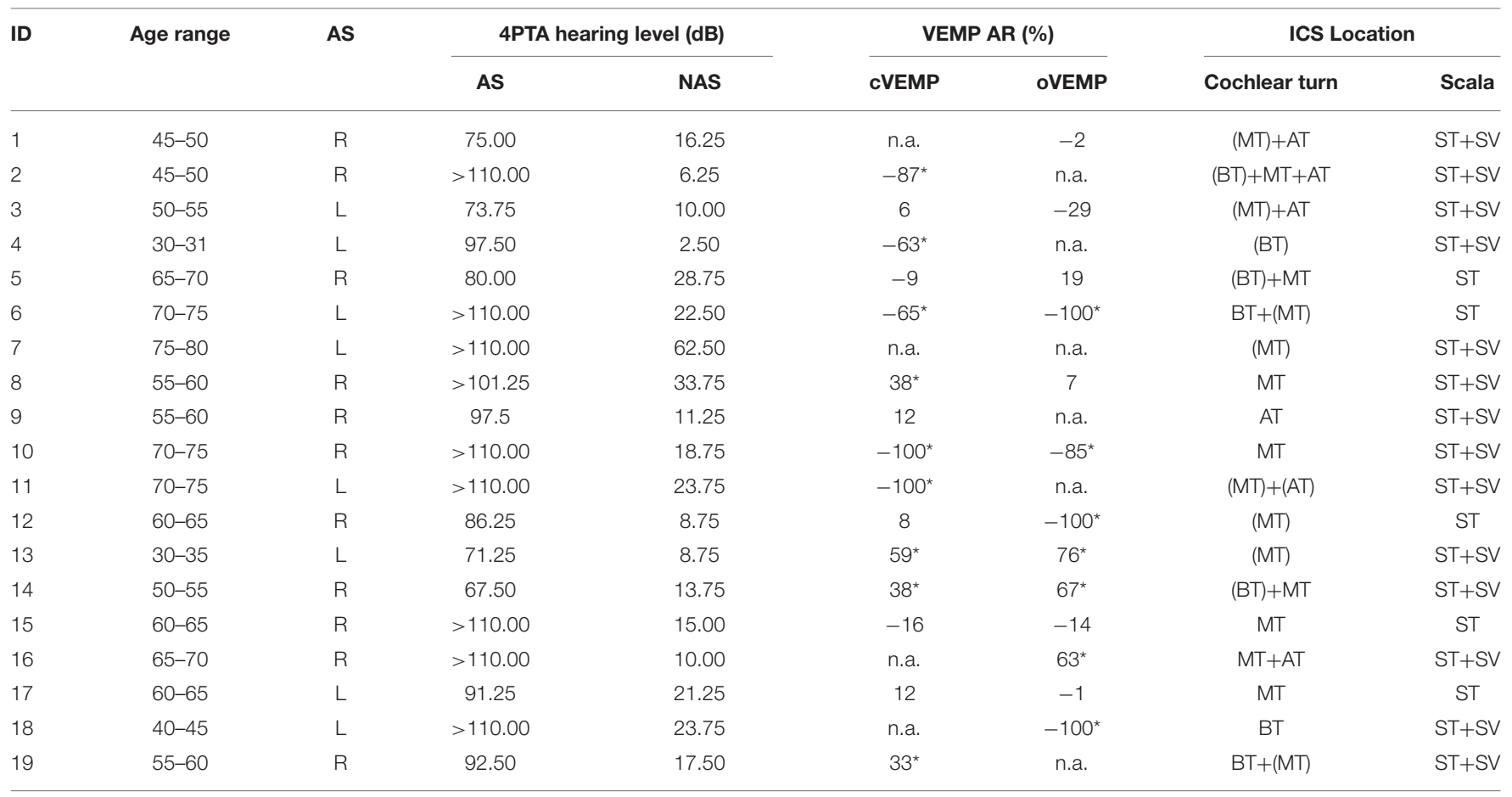

4PTA, pure tone average at 0.5, 1, 3, 4 kHz; AS, affected side; NAS, non-affected side; AR, asymmetry ratio; ICS, intracochlear schwannoma; BT, basal turn; MT, middle turn; AT, apical turn; ST, scala tumpani; SV, scala vestibuli; (, partially; *abnormal AR.

and did not show any signs of a third mobile window of the otic capsule.

The analysis of enhanced VEMPs and tumor localization showed that in 2 of the 5 patients with enhanced VEMPs only the MT (\#8,\#13), in 2 patients the BT and MT (\#14,\#19), and in 1 patient MT and AT (\#16) was involved. A correlation of enhanced VEMPs and tumor localization with respect to the cochlear turn could therefore not be observed. With respect to the scala, the tumors were located in both, ST and SV, in all five patients with enhanced VEMPs and in none of the patients where only the ST was affected by the tumor.

\section{DISCUSSION}

Only a few studies reported VEMP results in patients with intralabyrinthine schwannoma (ILS) and mostly described absent or decreased cVEMPs and/or oVEMPs in these patients $(6,11$, 12). The exact tumor localization and description of VEMP results in patients with solely intracochlear schwannomas (ICS) was done by Dubernard et al. (6) for 12 patients. Cervical VEMPs were absent or significantly reduced in $50 \%$ of the patients and "preserved" in the remaining 50\%. Patients with intravestibular tumors were also examined in their study and were-not surprisingly - found to have a higher rate of absent or reduced cVEMPs, which is likely due to a direct impact of the tumor mass on the otolith organs. The results from our study are the first to systematically analyze both oVEMPs and cVEMPs in a series of cases with solely intracochlear tumors.
Despite the intracochlear position, the tumor affected the vestibular response which was found to be absent, reduced and in some cases enhanced. We observed absent or reduced cVEMPs in $47 \%$ of the patients and normal cVEMPs in $32 \%$. This is in line with the results described by Dubernard et al. (6). The surprising result was that the cVEMPs were enhanced in $21 \%$ of the patients in our study. Ocular VEMPs were reduced or absent in 53\% and normal in 32\%. Enhanced oVEMPs were observed in $15 \%$ of the patients.

Many factors can cause reduced or absent VEMP responses. Particularly in central pathology, the VEMPs are absent, reduced and/or prolonged which can be an early indicator of pathology (53-56). Many studies reported reduced or absent VEMPs in patients with cochleovestibular schwannoma (i.e., vestibular schwannoma, see introduction) and reported a strong relationship with tumor size (57). Cervical VEMPs in 38 ears of Neurofibromatosis Type 2 patients with small cochleovestibular schwannomas were described by Holliday et al. (58). Normal results were found in $71 \%$ and abnormal cVEMPs were found in $29 \%$ of the patients with a correlation between abnormal cVEMPs and tumor size. VEMP asymmetry ratios (ARs) in patients with cochleovestibular schwannomas can also be used as a screening tool for assessing the function of the superior and inferior vestibular nerves before and after surgical intervention (10). VEMP abnormalities in these patients are attributed to compressional and neurotoxic effects on the nerve and reduced vascular supply of the labyrinth. However, retrocochlear pathology or direct impact of the tumor on the otoliths was excluded in our study by 

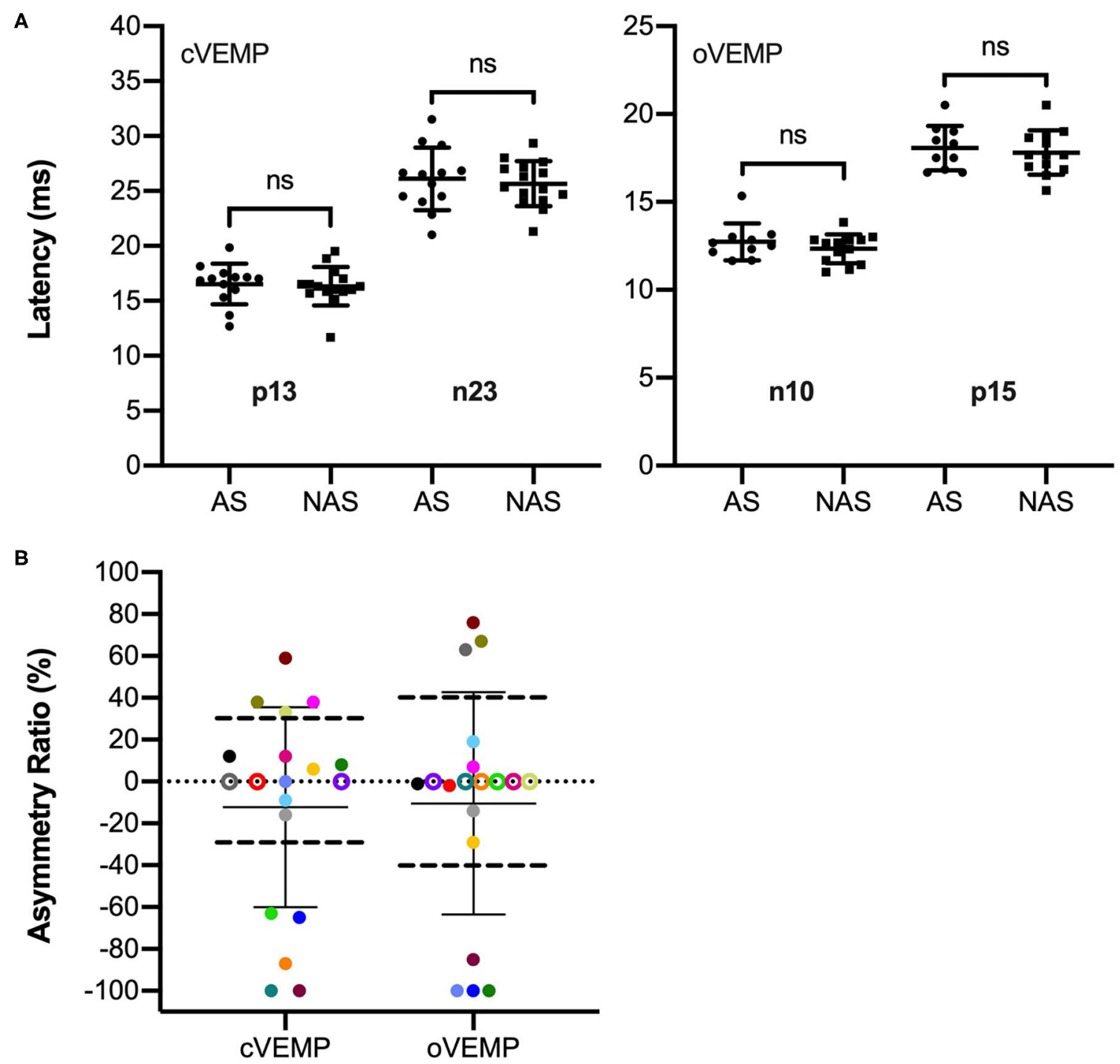

FIGURE 1 | Latency and VEMP asymmetry ratio (AR) results of the included patients $(n=19)$. (A) Response latencies with means and standard deviations for the cVEMP p13 and n23 for the side affected by the tumor (AS) $(n=13)$ and the non-affected side (NAS) $(n=16)$ as well as oVEMP n10 and p15 latencies for the AS $(n=10)$ and NAS $(n=13)$. No significant differences were found between latencies of the AS and NAS. (B) Signed ARs for cVEMPs and oVEMPs with means and standard deviations. Patients are color coded as they contribute to both the oVEMP and cVEMP AR data. Negative values indicate larger responses on the NAS, positive values indicate larger responses on the AS. For cVEMPs, ARs exceeding $\pm 30 \%$ were considered abnormal. For oVEMPs, abnormal ARs were larger/smaller than $\pm 40 \%$. The limits are illustrated by horizontal dashed lines. Data points above the thresholds represent enhanced VEMPs with respect to the AS, data points below the thresholds represent reduced VEMPs in the AS. Patients with absent responses on the AS are shown at AR $=-100 \%$. For patients with absent responses on both AS and NAS, the ARs are illustrated at $A R=0 \%$ as empty circles.

including only patients with solely intracochlear tumors. In addition, another patient with superior canal dehiscence was excluded, which could have acted as a confounding factor in the VEMP analysis. The pathophysiology of ICS leading to abnormal VEMPs is unknown and can only be speculated about. In patients with cochleovestibular schwannomas (without intracochlear localization of tumors), it has been reported that sensorineural hearing loss is associated with tumorsecreted factors containing pro-inflammatory cytokines which cause cochlear damage $(59,60)$. This could explain why large cochleovestibular schwannomas sometimes do not cause hearing loss while small ones do. This has not been investigated yet for ICS associated loss of otolith function but could be a similar mechanism. Possibly, the finding of reduced or absent VEMPs in these patients is attributed to a local cytotoxic effect conveyed by the labyrinthine fluids (6).

In the present study, the major and unexpected finding was that the VEMPs in ICS in some patients were enhanced but no latency prolongation was observed. Enhanced VEMPs are commonly seen in patients with third window syndrome. Thus, VEMPs have become a widely used tool in the diagnosis of third window syndrome $(16,24,29-33)$ and are enhanced in those 


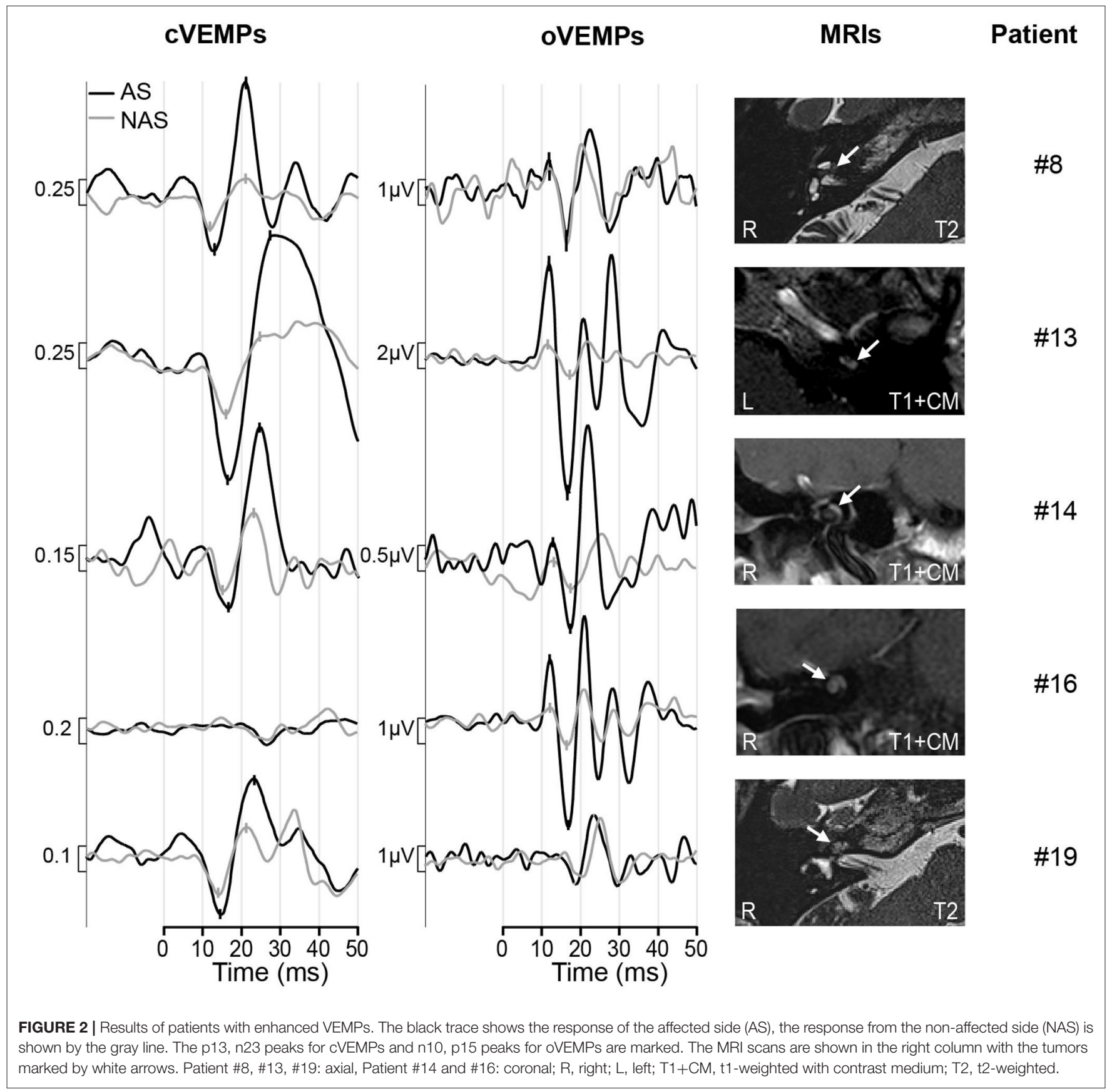

ears with a defect in the otic capsule (25-28). However, other conditions with endolymphatic hydrops such as Menière's disease have been shown to mimic third window syndrome showing reduced VEMP thresholds and enhanced amplitudes (44-47). To date, it was unknown that-with respect to VEMP resultsother inner ear disorders, ICS in particular, have the potential to mimic third window syndrome as well. In our experience, management of patients with ILS is highly individual and detailed functional evaluation of the vestibular labyrinth is important for counseling these patients regarding treatment options (especially with respect to surgical tumor removal) and outcome predictions $(61,62)$.
To explain the cause of enhanced VEMP amplitudes in ICS patients, we assume that this is due to a change of inner ear (fluid) mechanics caused by the tumor. In this study, we only included ICS patients to avoid bias due to direct influence of the tumor on the otoliths (e.g., as in intravestibular or intravestibulocochlear schwannomas) or by tumor in the internal auditory canal. Measurements, models, and animal studies have shown that in patients with superior semicircular canal dehiscence, a third mobile window leads to larger fluid displacement in the semicircular canals which activates canal neurons contributing to the VEMP and enhancing it $(34,37,63)$. This shows that mechanical changes cause enhanced VEMPs. 
It also supports the theory that mechanical changes can lead to enhanced VEMPs in patients with endolymphatic hydrops. The exact mechanisms of this observation are yet unknown. Endolymphatic hydrops can have various causes $(39,40)$. It seems possible that an obstructive tumor mass like an ICS has the potential to cause or act similar to endolymphatic hydrops. This idea is supported by the observation in our study that cVEMPs or oVEMPs were only enhanced, if both scala tympani (ST) and scala vestibuli (SV) were "blocked" by the tumor. This leads to the somehow contradictory observation that-with respect to VEMPs - a "third window syndrome" can act similar to a "minus 1 window syndrome" or "one window syndrome," when the cochlear is "blocked" by a tumor.

In such situations, acoustic stimulation cannot lead to a fully developed traveling wave within the cochlea. Nevertheless, the stapes displacement must still be compensated by a reciprocal displacement of the round window membrane. It is conceivable that this may lead to a perilymph flow which is oscillating more or less directly between the oval window and the round window including a corresponding displacement of the basal basilar membrane. Clearly, in this configuration the fluid dynamics in the basal region of the cochlea would be significantly altered and-similar to the third window syndrome (36) - there would be higher flow velocities close to the saccule which may lead to enhanced VEMPs. It appears also possible that the cochlear blockage leads to a suppression of the piston-like stapes motion (PSM) and that the acoustic stimulation leads only to a "rocking stapes motion" (RSM) which does not create any net fluid displacement within the otic capsule. It has been shown [Figure 5 in Edom et al. (64)] that RSM leads to significantly increased perilymph flow in the basal region of the cochlea which may affect saccular stimulation and be connected to enhanced VEMPs. Computer-modeling of the fluid dynamics of a "blocked" cochlea may have the potential to give answers to the question if the mass effect without concomitant neural damage could cause the enhancement of VEMPs and should be considered in future studies.

Limitations of the study include its retrospective design, which is due to the nature of the observation which was more or less accidental. This is also a reason, why the study did not include threshold measurements. Since VEMPs evoked by bone conducted vibration were not available at that time, only air conduction was used for stimulation. While any third window abnormalities were excluded in most patients, CT scans were not available in 1 of the 5 patients with enhanced VEMPs, and CTs (although thin-sliced) were technically not targeted specifically on exclusion of bony defects of the inner ear. These limitations can be addressed in further studies including specific history taking (i.e., checklists for symptoms of third window syndromes), threshold measurements in all patients with enhanced VEMPs as well as specific CT scans in these patients. Another aspect which has to be considered in further studies is the evolution of VEMPs in these patients. It should be examined, how the VEMP amplitudes and latencies change over time, possibly in the course of tumor growth. Regarding the different outcomes, i.e., especially reduced or absent in contrast to normal or enhanced VEMPs, the tumors' intrinsic biology with respect to tumor secreted factors should be investigated as was done for cochleovestibular schwannomas causing hearing loss $(59,60)$. This is important to assess the clinical relevance of normal, absent or reduced, and enhanced VEMPs and might become beneficial for counseling ICS patients.

\section{CONCLUSION}

We described that enhanced VEMP amplitudes could be observed in patients with intracochlear schwannoma. It was an unexpected novelty that in addition to conditions described by the general term of the third window syndrome, or in Menière's disease, VEMP amplitudes can be enhanced in patients with intracochlear schwannoma. Response latencies were not significantly different between the side affected by the tumor and the non-affected side. Intracochlear tumors should therefore be added to the list of conditions which may cause increased VEMP amplitudes. Since management of patients with intracochlear schwannomas is highly individual, these findings might become beneficial for counseling these patients regarding treatment options and outcome predictions.

\section{DATA AVAILABILITY STATEMENT}

The raw data supporting the conclusions of this article will be made available by the authors, without undue reservation.

\section{ETHICS STATEMENT}

The studies involving human participants were reviewed and approved by Ethics committee of the Medical Faculty of Martin Luther University Halle-Wittenberg and the University Hospital Halle. The patients/participants provided their written informed consent to participate in this study.

\section{AUTHOR CONTRIBUTIONS}

LF and SP contributed the conception and design of the study and organized the database. LF, SP, and SK performed the data analysis. TR contributed to the data analysis and the visualization of results. IC and DO made contributions with interpretation of the results and generation of hypotheses. LF wrote the first draft of the manuscript. All authors contributed to the manuscript revision, read, and approved the submitted version.

\section{FUNDING}

The authors acknowledge the financial support of the Open Access Publication Fund of the Martin Luther University Halle-Wittenberg.

\section{ACKNOWLEDGMENTS}

The authors thank Karl-Johan Fredén Jansson for support in acquiring data of patient $\# 8$ in this study and providing the data plots for this patient. 


\section{REFERENCES}

1. Merchant SN, McKenna MJ. Schwannoma. In: Merchant SN, Nadol JB, editors. Schuknecht's Pathology of the Ear. 3rd ed. Shelton: People's medical publishing house (2010). p. 492-510.

2. Kennedy RJ, Shelton C, Salzman KL, Davidson HC, Harnsberger HR. Intralabyrinthine schwannomas: diagnosis, management, and a new classification system. Otol Neurotol. (2004) 25:1607. doi: 10.1097/00129492-200403000-00014

3. Slattery EL, Babu SC, Chole RA, Zappia JJ. Intralabyrinthine schwannomas mimic cochleovestibular disease: symptoms from tumor mass effect in the labyrinth. Otol Neurotol. (2014) 36:167-71. doi: 10.1097/MAO.0000000000000516

4. Tieleman A, Casselman JW, Somers T, Delanote J, Kuhweide R, Ghekiere J, et al. imaging of intralabyrinthine schwannomas: a retrospective study of 52 cases with emphasis on lesion growth. Am J Neuroradiol. (2008) 29:898905. doi: 10.3174/ajnr.A1026

5. Van Abel KM, Carlson ML, Link MJ, Neff BA, Beatty CW, Lohse CM, et al. Primary inner ear schwannomas: a case series and systematic review of the literature: primary inner ear schwannomas. Laryngoscope. (2013) 123:195766. doi: 10.1002/lary.23928

6. Dubernard X, Somers T, Veros K, Vincent C. Clinical presentation of intralabyrinthine schwannomas: a multicenter study of 110 cases. Otol Neurotol. (2014) 35:1641-9. doi: 10.1097/MAO.00000000000 00415

7. Jerin C, Krause E, Ertl-Wagner B, Gürkov R. Clinical features of delayed endolymphatic hydrops and intralabyrinthine schwannoma: an imagingconfirmed comparative case series. English version. HNO. (2017) 65:415. doi: 10.1007/s00106-016-0199-6

8. Plontke SK, Rahne T, Pfister M, Götze G, Heider C, Pazaitis $\mathrm{N}$, et al. Intralabyrinthine schwannomas: surgical management and hearing rehabilitation with cochlear implants. HNO. (2017) 65:136-48. doi: 10.1007/s00106-017-0364-6

9. Salzman KL, Childs AM, Davidson HC, Kennedy RJ, Shelton C, Harnsberger HR. Intralabyrinthine schwannomas: imaging diagnosis and classification. Am J Neuroradiol. (2012) 33:104-9. doi: 10.3174/ajnr.A2712

10. Rahne T, Plößl S, Plontke SK, Strauss C. Preoperative determination of nerve of origin in patients with vestibular schwannoma. HNO. (2018) 66:1621. doi: 10.1007/s00106-017-0416-y

11. Lee SU, Bae YJ, Kim HJ, Choi JY, Song JJ, Choi BY, et al. Intralabyrinthine schwannoma: distinct features for differential diagnosis. Front Neurol. (2019) 10:750. doi: 10.3389/fneur.2019.00750

12. Ralli M, Nola G, Fusconi M, Sparvoli L, Ralli G. ocular vestibular evoked myogenic potentials and intravestibular intralabyrinthine schwannomas. Ear Nose Throat J. (2018) 97:E21-5. doi: 10.1177/014556131809700703

13. Minor LB, Solomon D, Zinreich JS, Zee DS. Sound- and/or pressure-induced vertigo due to bone dehiscence of the superior semicircular canal. Arch Otolaryngol Neck Surg. (1998) 124:249-58. doi: 10.1001/archotol.124.3.249

14. Young L, Isaacson B. Cochlear and petrous carotid canal erosion secondary to cholesteatoma. Otol Neurotol. (2009) 31:697-8. doi: 10.1097/MAO.0b013e31819bd803

15. Park JJH, Shen A, Loberg C, Westhofen M. The relationship between jugular bulb position and jugular bulb related inner ear dehiscence: a retrospective analysis. Am J Otolaryngol. (2015) 36:347-51. doi: 10.1016/j.amjoto.2014.12.006

16. Gopen Q, Zhou G, Poe D, Kenna M, Jones D. Posterior semicircular canal dehiscence: first reported case series. Otol Neurotol. (2010) 31:33944. doi: 10.1097/MAO.0b013e3181be65a4

17. Blake DM, Tomovic S, Vazquez A, Lee H, Jyung RW. Cochlear-facial dehiscence-A newly described entity. Laryngoscope. (2014) 124:2839. doi: 10.1002/lary.24223

18. Fujita T, Kobayashi T, Saito K, Seo T, Ikezono T, Doi K. Vestibulemiddle ear dehiscence tested with perilymph-specific protein Cochlin-Tomoprotein (CTP) detection test. Front Neurol. (2019) 10:47. doi: 10.3389/fneur.2019.00047

19. Manzari L. Multiple dehiscences of bony labyrinthine capsule. A rare case report and review of the literature. Acta Otorhinolaryngol Ital. (2010) 30:31720. doi: 10.3233/VES-140517
20. Manzari L, Scagnelli P. Large bilateral internal auditory meatus associated with bilateral superior semicircular canal dehiscence. Ear Nose Throat J. (2013) 92:25-33. doi: 10.1177/014556131309200109

21. Koo JW, Hong SK, Kim DK, Kim JS. Superior semicircular canal dehiscence syndrome by the superior petrosal sinus. J Neurol Neurosurg Psychiatry. (2010) 81:465-7. doi: 10.1136/jnnp.2008.155564

22. Ionescu EC, Al Tamami N, Neagu A, Ltaief-Boudrigua A, Gallego S, Hermann $\mathrm{R}$, et al. superior semicircular canal ampullae dehiscence as part of the spectrum of the third window abnormalities: a case study. Front Neurol. (2017) 8:683. doi: 10.3389/fneur.2017.00683

23. Ho ML, Moonis G, Halpin CF, Curtin HD. Spectrum of third window abnormalities: semicircular canal dehiscence and beyond. Am J Neuroradiol. (2017) 38:2-9. doi: 10.3174/ajnr.A4922

24. Wackym PA, Balaban CD, Zhang P, Siker DA, Hundal JS. Third window syndrome: surgical management of cochlea-facial nerve dehiscence. Front Neurol. (2019) 10:1281. doi: 10.3389/fneur.2019.01281

25. Minor LB. Clinical manifestations of superior semicircular canal dehiscence. Laryngoscope. (2005) 115:1717-27. doi: 10.1097/01.mlg.0000178324.55729.b7

26. Verrecchia L, Westin M, Duan M, Brantberg K. Ocular vestibular evoked myogenic potentials to vertex low frequency vibration as a diagnostic test for superior canal dehiscence. Clin Neurophysiol. (2016) 127:21349. doi: 10.1016/j.clinph.2016.01.001

27. Manzari L, Burgess AM, McGarvie LA, Curthoys IS. Ocular and cervical vestibular evoked myogenic potentials to $500 \mathrm{~Hz} F z$ bone-conducted vibration in superior semicircular canal dehiscence. Ear Hear. (2012) 33:50820. doi: 10.1097/AUD.0b013e3182498c09

28. Govender S, Fernando T, Dennis DL, Welgampola MS, Colebatch JG. Properties of $500 \mathrm{~Hz}$ air- and bone-conducted vestibular evoked myogenic potentials (VEMPs) in superior canal dehiscence. Clin Neurophysiol. (2016) 127:2522-31. doi: 10.1016/j.clinph.2016.02.019

29. Aw ST, Welgampola MS, Bradshaw AP, Todd MJ, Magnussen JS, Halmagyi GM. Click-evoked vestibulo-ocular reflex distinguishes posterior from superior canal dehiscence. Neurology. (2010) 75:933-5. doi: 10.1212/WNL.0b013e3181f11dfd

30. Taylor RL, Bradshaw AP, Magnussen JS, Gibson WPR, Halmagyi GM, Welgampola MS. Augmented ocular vestibular evoked myogenic potentials to air-conducted sound in large vestibular aqueduct syndrome. Ear Hear. (2012) 33:768-71. doi: 10.1097/AUD.0b013e31825ce613

31. Modugno GC, Magnani G, Brandolini C, Savastio G, Pirodda A. Could vestibular evoked myogenic potentials (VEMPs) also be useful in the diagnosis of perilymphatic fistula? Eur Arch Otorhinolaryngol. (2006) 263:5525. doi: 10.1007/s00405-006-0008-z

32. Friedmann DR. A clinical and histopathologic study of jugular bulb abnormalities. Arch Otolaryngol Neck Surg. (2012) 138:6671. doi: 10.1001/archoto.2011.231

33. Schneiders SMD, Rainsbury JW, Hensen EF, Irving RM. Superior petrosal sinus causing superior canal dehiscence syndrome. J Laryngol Otol. (2017) 131:593-7. doi: 10.1017/S0022215117001013

34. Rosowski JJ, Songer JE, Nakajima HH, Brinsko KM, Merchant SN. Clinical, experimental, and theoretical investigations of the effect of superior semicircular canal dehiscence on hearing mechanisms. Otol Neurotol. (2004) 25:323-32. doi: 10.1097/00129492-200405000-00021

35. Iversen MM, Zhu H, Zhou W, Della Santina CC, Carey JP, Rabbitt RD. Sound abnormally stimulates the vestibular system in canal dehiscence syndrome by generating pathological fluid-mechanical waves. Sci Rep. (2018) 8:10257. doi: 10.1038/s41598-018-28592-7

36. Grieser BJ, Kleiser L, Obrist D. Identifying mechanisms behind the tullio phenomenon: a computational study based on first principles. J Assoc Res Otolaryngol. (2016) 17:103-18. doi: 10.1007/s10162-016-0553-0

37. Curthoys IS. The new vestibular stimuli: sound and vibration-anatomical, physiological and clinical evidence. Exp Brain Res. (2017) 235:95772. doi: 10.1007/s00221-017-4874-y

38. Curthoys IS, Grant JW, Burgess AM, Pastras CJ, Brown DJ, Manzari L. Otolithic receptor mechanisms for vestibular-evoked myogenic potentials: a review. Front Neurol. (2018) 9:366. doi: 10.3389/fneur.2018.00366

39. Ferster APO, Cureoglu S, Keskin N, Paparella MM, Isildak H. Secondary endolymphatic hydrops. Otol Neurotol. (2017) 38:774-9. doi: 10.1097/MAO.0000000000001377 
40. Smeds H, Eastwood HT, Hampson AJ, Sale P, Campbell LJ, Arhatari BD, et al. Endolymphatic hydrops is prevalent in the first weeks following cochlear implantation. Hear Res. (2015) 327:48-57. doi: 10.1016/j.heares. 2015. 04.017

41. Sufyan Amir Paisal M, Azmi Wahab M, Taib I, Mat Isa N, Ramli Y, Md Seri S, et al. Flow behaviour in normal and Meniere's disease of endolymphatic fluid inside the inner ear. IOP Conf Ser Mater Sci Eng. (2017) 243:012033. doi: 10.1088/1757-899X/243/1/012033

42. Grieser BJ, McGarvie LA, Kleiser L, Manzari L, Obrist D, Curthoys IS. Numerical investigations of the effects of endolymphatic hydrops on the VOR response. J Vestib Res. (2014) 24:219.

43. Obrist D. Flow phenomena in the inner ear. Annu Rev Fluid Mech. (2019) 51:487-510. doi: 10.1146/annurev-fluid-010518-040454

44. Young YH, Wu CC, Wu CH. Augmentation of vestibular evoked myogenic potentials: an indication for distended saccular hydrops. Laryngoscope. (2002) 112:509-12. doi: 10.1097/00005537-200203000-00019

45. Taylor RL, Zagami AS, Gibson WP, Black DA, Watson SR, Halmagyi MG, et al. Vestibular evoked myogenic potentials to sound and vibration: characteristics in vestibular migraine that enable separation from Menière's disease. Cephalalgia. (2012) 32:213-25. doi: 10.1177/03331024114 34166

46. Wen MH, Cheng PW, Young YH. Augmentation of ocular vestibular-evoked myogenic potentials via bone-conducted vibration stimuli in ménière disease. Otolaryngol Neck Surg. (2012) 146:797-803. doi: 10.1177/0194599811433982

47. Manzari L, Tedesco AR, Burgess AM, Curthoys IS. Ocular and cervical vestibular-evoked myogenic potentials to bone conducted vibration in Ménière's disease during quiescence vs during acute attacks. Clin Neurophysiol. (2010) 121:1092-101. doi: 10.1016/j.clinph.2010. 02.003

48. Rahne T, Weiser C, Plontke S. Neurofeedback-Controlled comparison of the head elevation versus head rotation and head-hand methods in eliciting cervical vestibular evoked myogenic potentials. Audiol Neurotol. (2014) 19:327-35. doi: 10.1159/000362661

49. McCaslin DL, Fowler A, Jacobson GP. Amplitude normalization reduces Cervical Vestibular Evoked Myogenic Potential (cVEMP) amplitude asymmetries in normal subjects: proof of concept. J Am Acad Audiol. (2014) 25:268-77. doi: 10.3766/jaaa.25.3.6

50. Welgampola MS, Colebatch JG. Characteristics and clinical applications of vestibular-evoked myogenic potentials. Neurology. (2005) 64:16828. doi: 10.1212/01.WNL.0000161876.20552.AA

51. Govender S, Rosengren SM, Colebatch JG. Vestibular neuritis has selective effects on air- and bone-conducted cervical and ocular vestibular evoked myogenic potentials. Clin Neurophysiol. (2011) 122:1246-55. doi: 10.1016/j.clinph.2010.12.040

52. Koo TK, Li MY. A guideline of selecting and reporting intraclass correlation coefficients for reliability research. J Chiropr Med. (2016) 15:15563. doi: $10.1016 /$ j.jcm.2016.02.012

53. Oh SY, Kim JS, Lee JM, Shin BS, Hwang SB, Kwak KC, et al. Ocular vestibular evoked myogenic potentials induced by air-conducted sound in patients with acute brainstem lesions. Clin Neurophysiol. (2013) 124:7708. doi: 10.1016/j.clinph.2012.09.026
54. Skorić MK, Adamec I, Madarić VN, Habek M. Evaluation of brainstem involvement in multiple sclerosis. Can J Neurol Sci. (2014) 41:3469. doi: $10.1017 / \mathrm{S} 0317167100017285$

55. Oh SY, Kim HJ, Kim JS. Vestibular-evoked myogenic potentials in central vestibular disorders. J Neurol. (2016) 263:21020. doi: $10.1007 /$ s00415-015-7860-y

56. Di Stadio A, Dipietro L, Ralli M, Greco A, Ricci G, Bernitsas E. The role of vestibular evoked myogenic potentials in multiple sclerosis-related vertigo. A systematic review of the literature. Mult Scler Relat Disord. (2019) 28:15964. doi: 10.1016/j.msard.2018.12.031

57. Taylor RL, Kong J, Flanagan S, Pogson J, Croxson G, Pohl D, et al. Prevalence of vestibular dysfunction in patients with vestibular schwannoma using video head-impulses and vestibular-evoked potentials. J Neurol. (2015) 262:122837. doi: 10.1007/s00415-015-7697-4

58. Holliday MA, Kim HJ, Zalewski CK, Wafa T, Dewan R, King KA, et al. Audiovestibular characteristics of small cochleovestibular schwannomas in neurofibromatosis Type 2. Otolaryngol Neck Surg. (2014) 151:11724. doi: 10.1177/0194599814529081

59. Soares VYR, Atai NA, Fujita T, Dilwali S, Sivaraman S, Landegger LD, et al. Extracellular vesicles derived from human vestibular schwannomas associated with poor hearing damage cochlear cells. Neuro-Oncol. (2016) 18:1498-507. doi: 10.1093/neuonc/now099

60. Sagers JE, Sahin MI, Moon I, Ahmed SG, Stemmer-Rachamimov A, Brenner GJ, et al. NLRP3 inflammasome activation in human vestibular schwannoma: implications for tumor-induced hearing loss. Hear Res. (2019) 381:107770. doi: 10.1016/j.heares.2019.07.007

61. Plontke SK, Kösling S, Rahne T. Cochlear implantation after partial or subtotal cochleoectomy for intracochlear schwannoma removal-a technical report. Otol Neurotol. (2018) 39:365-71. doi: 10.1097/MAO.0000000000001696

62. Plontke SK, Fröhlich L, Wagner L, Kösling S, Götze G, Siebolts U, et al. How much cochlea do you need for cochlear implantation? Otol Neurotol. (2020) 41:694-703. doi: 10.1097/MAO.0000000000002614

63. Songer JE, Rosowski JJ. The effect of superior-canal opening on middle-ear input admittance and air-conducted stapes velocity in chinchilla. J Acoust Soc Am. (2006) 120:258-69. doi: 10.1121/1.2204356

64. Edom E, Obrist D, Henniger R, Kleiser L, Sim JH, Huber AM. The effect of rocking stapes motions on the cochlear fluid flow and on the basilar membrane motion. J Acoust Soc Am. (2013) 134:374958. doi: $10.1121 / 1.4824159$

Conflict of Interest: The authors declare that the research was conducted in the absence of any commercial or financial relationships that could be construed as a potential conflict of interest.

Copyright $\odot 2020$ Fröhlich, Curthoys, Kösling, Obrist, Rahne and Plontke. This is an open-access article distributed under the terms of the Creative Commons Attribution License (CC BY). The use, distribution or reproduction in other forums is permitted, provided the original author(s) and the copyright owner(s) are credited and that the original publication in this journal is cited, in accordance with accepted academic practice. No use, distribution or reproduction is permitted which does not comply with these terms. 